\title{
Precision Radiotherapy for Small Animal Research $^{\star}$
}

\author{
Mohammad Matinfar ${ }^{1}$, Iulian Iordachita ${ }^{1}$, Eric Ford ${ }^{2}$, John Wong $^{2}$, \\ and Peter Kazanzides ${ }^{1}$
}

${ }^{1}$ Dept. of Computer Science, Johns Hopkins University, Baltimore, MD

${ }^{2}$ Dept. of Radiation Oncology and Molecular Radiation Sciences, Johns Hopkins Medical Institution, Baltimore, MD

\begin{abstract}
Preclinical research using well characterized small animal models has provided tremendous benefits to medical research, enabling low cost, large scale trials with high statistical significance of observed effects. The goal of the Small Animal Radiation Research Platform (SARRP) is to make those models available for the development and evaluation of novel radiation therapies. SARRP demonstrates the capabilities of delivering high resolution, sub-millimeter, optimally planned conformal radiation with on-board cone-beam $\mathrm{CT}$ (CBCT) guidance. The system requires accurate calibration of the x-ray beam for both imaging and radiation treatment. In this paper, we present a novel technique using an $\mathrm{x}$-ray camera for calibration of the treatment beam. This technique does not require precise positioning or calibration of the x-ray camera.
\end{abstract}

\section{Introduction}

It is desirable to test new radiotherapy techniques in a small animal model prior to clinical deployment. Although many mouse models of human cancer are currently available, existing imaging and therapeutic systems are ill suited to such small subjects, and the equipment is also in high demand and seldom available for lengthy laboratory trials. DesRosiers [1 notes that most clinical systems, such as a linear accelerator (Linac), have an accuracy of about $\pm 2 \mathrm{~mm}$, whereas small animals (with correspondingly smaller structures) require an accuracy that is an order of magnitude higher (e.g., $\pm 0.2 \mathrm{~mm}$ ). She demonstrated the feasibility of using a Gamma Knife, with an accuracy of about $\pm 0.5 \mathrm{~mm}$, for small animal research. The disadvantage of the Gamma Knife, and the Gammacell often used for small animal research, is that radiation can only be delivered as single beams. In contrast, systems such as the Linac can deliver radiation over a conformal arc.

The SARRP aims to address these issues by providing a platform that can perform high-resolution imaging and accurate conformal beam therapy on standard animal models for human cancers [2. It consists of a kilovoltage x-ray tube mounted on a rotating gantry. The tube provides a low-energy beam for

\footnotetext{
* This work was supported by NIH R01 CA108449.
} 
cone-beam CT imaging (with a flat panel detector) and a high-energy beam for radiotherapy. The animal is placed on a four-axis robotic positioner that provides rotary motion for cone-beam CT and translation and rotation for radiotherapy targeting. The SARRP offers several advantages over the Gammacell, including portability and the ability to deliver radiation over a conformal arc.

A similar effort is being undertaken by Stojadinovic [3], who notes that "a small animal conformal irradiator or a microradiation therapy (microRT) device is a missing link for studying response to therapeutic doses of radiation." $\mathrm{He}$ reported the results of simulation studies to validate his blueprints. Although construction details are not reported, one notable difference is that his system will use a radioisotope rather than a kilovoltage x-ray source.

By combining cone-beam $\mathrm{CT}$ imaging and radiotherapy, the SARRP offers capabilities that today generally require the use of separate clinical systems (e.g., a CT scanner and Linac). Therefore, the SARRP is keeping pace with new developments in human clinical systems, such as the integrated Linac and x-ray imaging system reported by Sharpe et al. 4 .

This paper presents an overview of the SARRP design (Section 2) and focuses on the calibration of the x-ray treatment system (Section 3), with results presented in Section 4. The calibration method uses an x-ray camera, and a visual servoing method, to measure and correct for errors in the position and orientation of the treatment beam. This method has some similarity to the calibration method of the Cyberknife system, which uses a light-sensitive crystal [5]. Their method uses a $2 \mathrm{~mm}$ laser beam that is assumed to be coaxial with the treatment beam.

\section{System Description}

The SARRP employs an isocentric design for imaging and irradiation (Fig. 1). The entire assembly is portable to facilitate ease in deployment. A dual-focus constant voltage $\mathrm{x}$-ray source operating up to $225 \mathrm{kVp}$ is mounted on a gantry

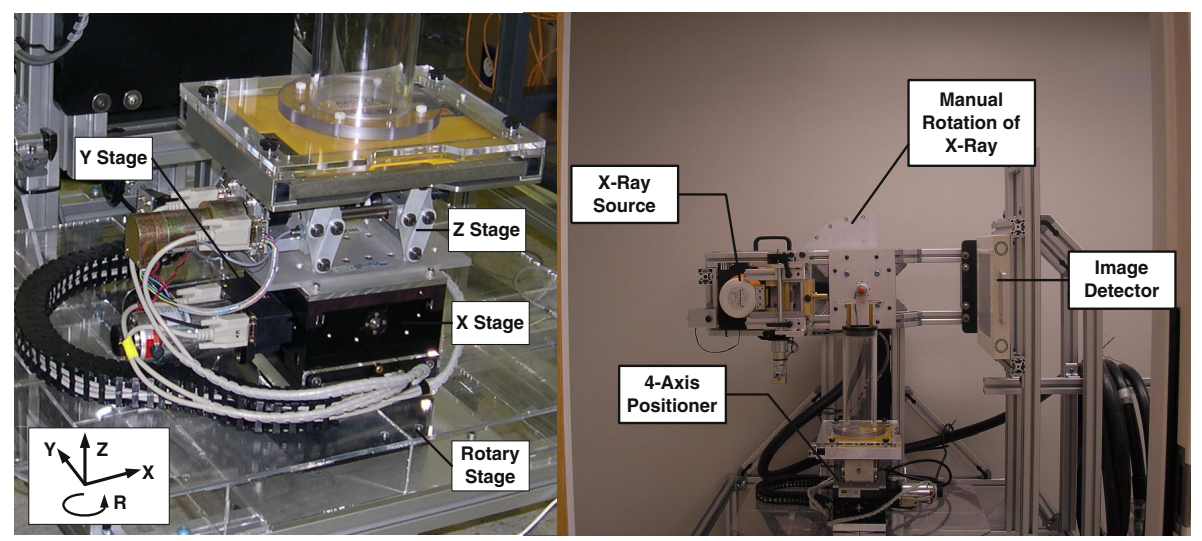

Fig. 1. Components of the Small Animal Radiation Research Platform 
with a nominal source-to-isocenter distance of $35 \mathrm{~cm}$. Gantry rotation is limited to $120^{\circ}$ from vertical at fixed $15^{\circ}$ increments. Computer controlled robotic translation and rotation stages are used to position the animal. A novel CBCT imaging method is devised where the prone animal is rotated between the stationary x-ray source and a two dimensional (2D) digital flat-panel detector in a horizontal setup. Radiation beams with dimensions ranging from $0.5 \mathrm{~mm}$ in diameter to $200 \times 200 \mathrm{~mm}$ are delivered with regularly and irregularly shaped collimators or blocks. Simple and complex three dimensional (3D) conformal dose distributions are delivered using a combination of gantry and robotic stage motions. Additional details can be found in 2 .

\section{Calibration of Sarrp}

Radiation treatment is often delivered from multiple poses that are intended to intersect at a specified point (the treatment isocenter). If the target (tumor) is positioned at this point, it will receive a higher dose than the surrounding healthy tissue. With the SARRP, rotation about a target can be obtained via the rotary axis $(\theta)$ or the x-ray gantry $(\phi)$. In reality, there is no single treatment isocenter because the two axes of rotation do not (in general) intersect and even if they did, physical factors such as gravity loading on the mechanical structure would cause the x-rays to deviate from their ideal positions. Thus, x-rays delivered from multiple gantry angles will not intersect at a single point. One common solution is to find a single "best-fit" isocenter, but this approach does not yield sufficient accuracy for our system. Our solution is to define nine different isocenters - one for each position of the x-ray gantry. This is particularly appropriate for the SARRP because the gantry is manually rotated to one of nine distinct orientations; more generally, for systems with continuous gantry rotation, this approach can be used at a finite set of angles and a complete solution can be obtained by model-fitting or interpolation. For convenience, we define the treatment isocenter to be the origin of the image coordinate system. Thus, the user need only position the target at the origin - the system will move it to the gantry-specific isocenter and will also adjust for any rotation $(\theta)$ of the target.

Fig. 2 depicts the two translations that are required to move the target from the image origin to the gantry-specific isocenter. It is assumed that the imaging system calibration (see [2]) aligns the image (CT) and robot coordinate systems, with the $Z$ axis defined by the axis of rotation $(\theta)$ and the origin lying along this axis (i.e.,

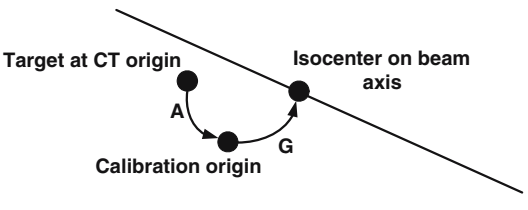

Fig. 2. Calibration overview $X=Y=0)$. We define the calibration origin as the point on the axis of rotation that is closest to the beam axis when the gantry is in the horizontal position $\left(\phi=0^{\circ}\right)$. Ideally, the translation $A$ from the image origin to the calibration origin should consist only of a $Z$ component (because the image origin should 
already lie on the axis of rotation), but in practice small $X$ and $Y$ values can compensate for inaccuracies in the image calibration. Second, the vector $G(\phi)$ translates from the calibration origin to the gantry-specific isocenter, which is obtained by measuring the lines that define the x-ray beams at each gantry angle $\phi$ (as discussed below) and then solving the following least-squares problem:

$$
\min \sum_{\phi}\|P(\phi)+D(\phi) s(\phi)-C\|^{2} \quad \phi=0^{\circ}, 15^{\circ}, \ldots
$$

where $P(\phi)$ and $D(\phi)$ are the (measured) point and direction vector that define the beam axis at the gantry angle $\phi, C$ is the (unknown) "best fit" isocenter, and $s(\phi)$ is the (unknown) parameter that defines the point on the line that is closest to $C$. Specifically, for each gantry angle $\phi$, the calibration offset $G(\phi)$ is given by $P(\phi)+D(\phi) s(\phi)$.

The translation vector $A$ (from image origin to calibration origin) is obtained by placing a ball bearing $(\mathrm{BB})$ on the robotic positioner and performing a CBCT scan. The BB is then moved to the origin of the image coordinate system and a collimator (e.g., $3 \times 3$ or $5 \times 5 \mathrm{~mm}$ ) is installed, with the gantry still at the horizontal (imaging) position. The robot $Z$ axis is adjusted until the BB appears vertically centered in the collimator, as measured on a $2 \mathrm{D}$ projection image. If desired, the robot $X$ and $Y$ axes can be adjusted to ensure that the BB does not move as the $\theta$ axis is rotated. The vector $A$ is given by the amount that the robot $X, Y$, and $Z$ axes were adjusted.

The calibration method for determining $G(\phi)$ (from calibration origin to gantry-specific isocenter) consists of a collimated beam (e.g., 1mm) and an x-ray camera, as shown in Fig. 3. This method does not require precise positioning or calibration of the x-ray camera. As a first step, we measure the axis of rotation of the robotic positioner because it is needed to determine the calibration origin. The measurement is performed by placing the x-ray gantry in the vertical position and rotating the stage through a set

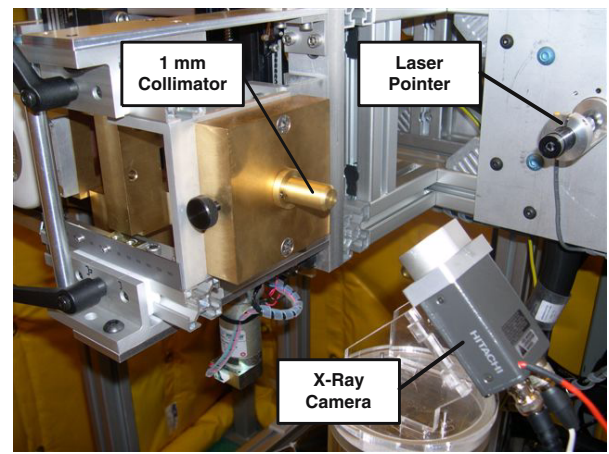

Fig. 3. Calibration using x-ray camera of angles, with $X=Y=0$ and $Z=Z_{0}$, where $Z_{0}$ is an arbitrary value that places the camera near the expected isocenter. The x-ray camera captures an image at each angle; these images are superimposed (added), as shown in Fig. 4 . The center of rotation, $C_{r}$ (in camera coordinates), is given by the center of gravity of the final image. Effectively, $C_{r}$ defines the coordinates of a specific camera pixel that we will use for all subsequent measurements. This pixel corresponds to the point $\left(0,0, Z_{0}\right)$, in robot coordinates, through which the axis of rotation passes. The axis of rotation is determined by moving the robot $Z$ axis to a second position, $Z_{1}$, and then moving the robot $X$ and $Y$ axes until the 


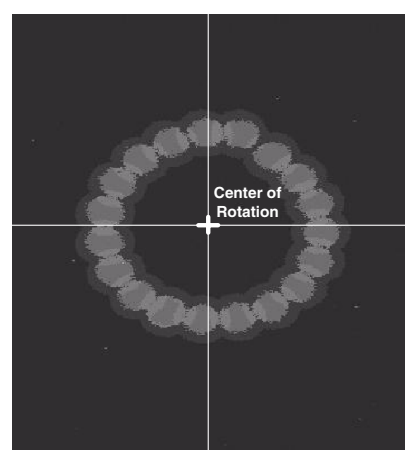

Fig. 4. Finding center of rotation
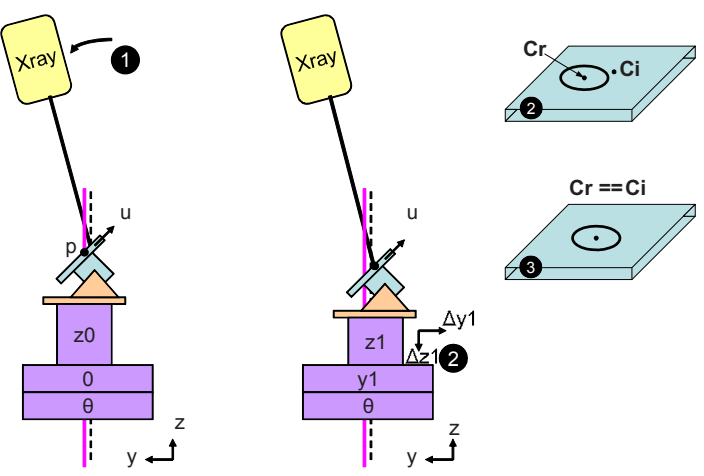

Fig. 5. Finding the x-ray beam

measured center of rotation, $C_{r 1}$, is equal to $C_{r}$. The axis of rotation is given by the vector $\left(\triangle x, \triangle y, Z_{1}-Z_{0}\right)$, where $\triangle x$ and $\triangle y$ are the amounts that the $X$ and $Y$ axes were moved, respectively. The intersection of the axis of rotation and the horizontal x-ray beam (to be measured below) define the calibration origin in robot coordinates.

Once $C_{r}$ is determined, we measure the line associated with the x-ray beam at each gantry angle as follows (see Fig. 5):

1. Move the x-ray gantry to the angle $\alpha$.

2. Capture an image with the x-ray camera and compute center of gravity, $C_{i}$.

3. Move the robot and capture images until $C_{i}$ is equal to $C_{r}$. The amount of $\mathrm{XYZ}$ motion provides the coordinates of one point on the x-ray beam, $P(\phi)$ in equation (11).

4. Move the robot to a new position and repeat the procedure. This provides a second point on the x-ray beam which, along with the first point, defines the direction of the x-ray beam, $D(\phi)$ in equation (11).

To perform the above procedure automatically, the controller should be able to read the images, find the appropriate feature in the image (e.g. center of gravity) and command the robot to move accordingly. This technique is often called visual servoing [6]. A block diagram representing the control method is shown in Fig. 6. It is necessary to calculate the image Jacobian inverse to convert errors in image coordinates $(u, v)$ to incremental robot motions $(d x, d y, d z)$. Any point on the x-ray line will produce an equilibrium position; this is not an issue because the calibration method can use any two points that lie on the line. We are currently using a precomputed image Jacobian that is based on our camera configuration. In particular, our x-ray camera is mounted at a $45^{\circ}$ angle. This enables us to keep the camera in the same position for gantry angles between $0^{\circ}$ and $90^{\circ}$ degrees, which is a requirement for our current calibration procedure (we are extending the method to handle the $-15^{\circ}$ and $-30^{\circ}$ gantry angles). Also, the camera is mounted so that the robot $x$ axis is approximately aligned with the camera $v$ axis. The robot $y$ and $z$ axes then affect only the camera $u$ axis, so it is 
straightforward to geometrically derive the non-trivial elements of the Jacobian, as shown in Fig. 6, when the x-ray gantry is positioned $\alpha^{\circ}$ from vertical:

$$
\frac{d u}{d y}=\frac{\tan (\alpha)}{\sin (45)(1+\tan (\alpha))}, \quad \frac{d u}{d z}=-\frac{\tan (90-\alpha)}{\sin (45)(1+\tan (90-\alpha))}
$$

Of course, our controller requires the inverse of this Jacobian. For this simple case, the inverse terms are given by:

$$
\frac{d y}{d u}=\frac{1}{K} \frac{d u}{d y}, \quad \frac{d z}{d u}=\frac{1}{K} \frac{d u}{d z} ; \quad K=\left(\frac{d u}{d y}\right)^{2}+\left(\frac{d u}{d z}\right)^{2}
$$

We found that controller performance is not very sensitive to the image Jacobian inverse, so our nominal matrix worked well in all practical situations. It is also possible to empirically estimate the image Jacobian by moving the robot a fixed distance along each axis and measuring the corresponding image change. This would require computation of a matrix pseudo-inverse to obtain the desired image Jacobian inverse. We obtained excellent performance from a simple proportional controller. Note that our controller inherently includes an integrator because we sum the incremental motions obtained by applying the image Jacobian inverse to the errors measured in image coordinates.
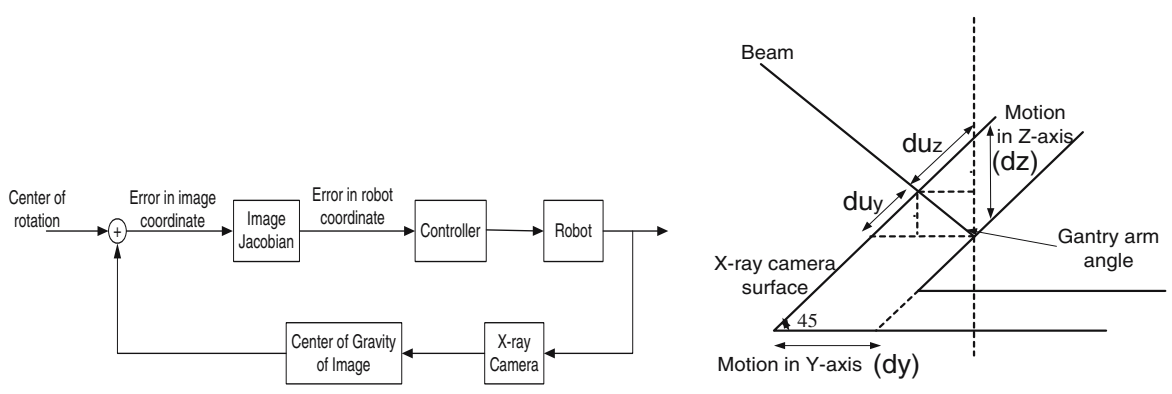

Fig. 6. Control block diagram of visual servo procedure, and relation between motion in robot $\mathrm{y}$ and $\mathrm{z}$-axes and image $u$ coordinate

\section{Results}

Based on the methods described in Section 3 , a set of experiments were carried out to find the different calibration offsets. The translation $A$ between the image origin and the calibration origin was found to be $(-0.4,-0.1,5.7)$. Note that the non-zero $X$ and $Y$ values indicate a small discrepancy from the axis of rotation measured during calibration of the imaging system. For the different gantry angles, the coordinates of a unit vector along the beam axis and a point on the beam axis were found. Fig. 7 shows these data when they are plotted in the YZplane (which is the plane spanned by the ideal beam axes). This corresponds to a digitally constructed "star shot" image. The offsets, $G(\phi)$, from the calibration origin to the gantry-specific isocenters are given in Table 1 . 


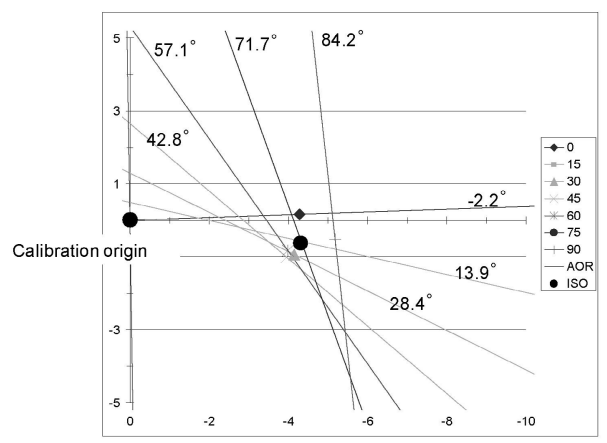

Fig. 7. Digitally constructed "star shot" showing x-ray beams for each gantry angle. Plot shows both nominal gantry angles (right) and measured angles (on each line).

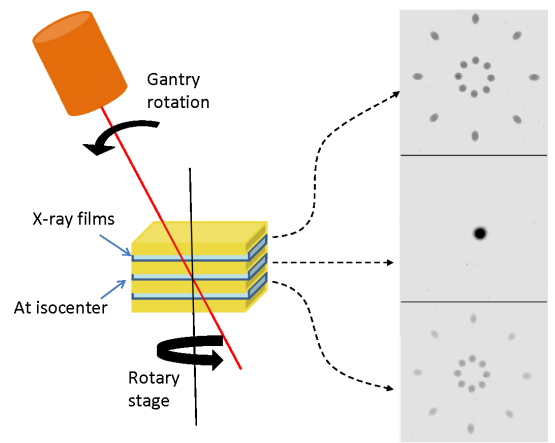

Fig. 8. Validation using radiographic films at gantry angles $(\phi)$ of $45^{\circ}$ and $75^{\circ}$, with $45^{\circ}$ stage rotations $(\theta)$
To validate the calibration results, we constructed a phantom with three radiographic films in a vertical stack, as shown in Fig. 8 We chose a target on the center film and delivered a collimated beam (1 $\mathrm{mm}$ diameter) from multiple gantry angles (45 and 75 degrees from horizontal) and at 45 degree increments of the rotary stage. The center film shows the spot where all x-rays intersect. The measured diameter of this spot is $1.73 \mathrm{~mm}$. We note that under ideal conditions, the diameter of the center spot should equal the major axis of the ellipse produced by the intersection of the 45 degree beam and the radiographic film. We can estimate the major axis by measuring it on the top film and projecting to the center film. We note that the collimator tip and target are approximately $300 \mathrm{~mm}$ and $350 \mathrm{~mm}$ from the source, respectively. The top film is about $5 \mathrm{~mm}$ above the center film, so a 45 degree beam would intersect it about $343 \mathrm{~mm}$ from the source, resulting in a magnification factor of $1.02(350 / 343)$ relative to the top film. On the top film, we measured the major axis to be $1.60 \mathrm{~mm}$, which magnifies to $1.63 \mathrm{~mm}$ on the center film. As a check, we note that a beam of diameter $D$ that intersects a horizontal plane at an angle $\phi$ produces an ellipse with a major axis of $D / \sin (\phi)$. The magnification between the collimator tip and the target is $1.17(350 / 300)$, so the diameter of the spot on the center film should be $1.17 / \sin \left(45^{\circ}\right)=1.65$ $\mathrm{mm}$, ignoring non-ideal effects such as those due to the penumbra. Nevertheless, this check confirms our estimate of an ideal spot diameter of $1.63 \mathrm{~mm}$, which is $0.10 \mathrm{~mm}$ less than the measured diameter. This result is promising, but further experiments are necessary to prove whether this high accuracy can be achieved repeatably and for any gantry angle $\phi$ and stage rotation $\theta$. 


\section{Conclusions and Future Work}

In this paper, we presented a novel system for small animal radiation with advanced imaging and irradiation capabilities that mimic methods employed in modern human treatment. The design incorporates three important components: focal irradiation of a target volume with sub-millimeter accuracy; on-board tomographic imaging; and conformal treatment planning to design more realistic pre-clinical irradiation experiments. The main focus of this paper is a novel technique for calibration of the treatment isocenter using an x-ray camera.

Future improvements include development of coordinated robot motion to achieve a virtual center of rotation for conformal therapy, rather than just the "step and shoot" approach shown in Fig. 8. Ongoing validation studies are focusing on characterizing the absolute accuracy of beam delivery. We are also constructing a second system with a motorized gantry.

\section{References}

1. DesRosiers, C., Mendonca, M., Tyree, C., Moskvin, V., Bank, M., Massaro, L., Bigsby, R., Caperall-Grant, A., Valluri, S., Dynlacht, J., Timmerman, R.: Use of the leksell gamma knife for localized small field lens irradiation in rodents. Tech. in Cancer Res. Treat. 2(5), 449-454 (2003)

2. Matinfar, M., Gray, O., Iordachita, I., Kennedy, C., Ford, E., Wong, J., Taylor, R., Kazanzides, P.: Small animal radiation research platform: Imaging, mechanics, control and calibration. In: Ayache, N., Ourselin, S., Maeder, A. (eds.) MICCAI 2007, Part I. LNCS, vol. 4791. Springer, Heidelberg (2007)

3. Stojadinovic, S., Low, D., Vicic, M., Mutic, S., Deasy, J., Hope, A., Parikh, P., Grigsby, P.: Progress toward a microradiation therapy small animal conformal irradiator. Medical Physics 33(10), 3834-3845 (2006)

4. Sharpe, M., Moseley, D., Purdie, T., Islam, M., Siewerdsen, J., Jaffray, D.: The stability of mechanical calibration for a kv cone beam computed tomography system integrated with linear accelerator. Medical Physics 33(1), 136-144 (2006)

5. Murphy, M.J., Cox, R.S.: The accuracy of dose localization for an image-guided frameless radiosurgery system. Medical Physics 23(12), 2043-2049 (1996)

6. Hutchinson, S., Hager, G.D., Corke, P.I.: A tutorial on visual servo control. IEEE Transactions on Robotics and Automation 12(5), 651-670 (1996) 